\title{
Simulation as a designed tool for material flow analysis by means of Witness Horizon
}

\author{
Tomáš Vysocký ${ }^{1,}$, Ludmila Nováková-Marcinčinová2 ${ }^{2}$ and Ema Nováková-Marcinčinováa \\ ${ }^{1}$ Faculty of manufacturing technologies with a seat in Prešov - Technical University in Košice, \\ Deparment of Computer aid of manufacturing technologies, 08001 Prešov, Slovakia \\ ${ }^{2}$ Faculty of manufacturing technologies with a seat in Prešov - Technical University in Košice, \\ Deparment of Computer aid of manufacturing technologies, 08001 Prešov, Slovakia \\ ${ }^{3}$ Faculty of manufacturing technologies with a seat in Prešov - Technical University in Košice, \\ Deparment of Computer aid of manufacturing technologies, 08001 Prešov, Slovakia
}

\begin{abstract}
In this post, the simulation is analysed, which positively influences the production process. Through simulation, it is possible to identify deficiencies found in different workplaces. Initially, the most familiar simulation software is used, which is used to create different types of simulations. The Witness system is described in more detail by simulation and subsequent results. The basic step before the simulation is to determine the data that enters the simulation. The workplace is composed of three basic workplaces that are contained in a number of machines. There is a conveyor between these workplaces, which ensures the transport of parts throughout the entire production hall. The result of this analysis is to evaluate the efficiency of each workplace and possible suggestions to increase the productivity of the entire process that are displayed using graphs.
\end{abstract}

\section{Introduction}

By means of computer simulation we prove create conditions of real experiment. We know follow phenomena, that may arise at exploring different elements. Most often, computer simulation is used in the industry, when is needed prevent mechanical destruction or improve quality of a manufacturing process. Manufacturing process is exposed to all risks, that may arise when its use. The main rules for creating simulation are right set conditions, which influence the whole simulation. By way of example may be mentioned simulation of torpedo with name REMUS, where has been verified this model of six-degrees of freedom [1].

The main elements of this simulation was the torpedo model, which had set center of gravity and coordination system in the middle point of the model. The experiment had to remind underwater environment, so environs had to remind water and all force, which

\footnotetext{
*Corresponding author: tomas.vysocky@tuke.sk
} 
acting on the solid part. Calculating was created though two methods Euler's method and method Runge-Kutta [1].

The basic tool for creating simulation is hardware and software equipment. For difficult simulation are needed the executive workstation, which have big processing power. In workstation must be mounted a high-capacity RAM, which can be assembled from more elements. Computer processor must contain more cores, compared with normal computer processors. In the case when simulation has a graphical character or result is complicated graphical output, that workstation must contain graphic card, which is determined for calculations. All components must be assembled all right and compatible. Therefore the workstation are bought as one component. In a few cases components are purchased as separate parts and assembled by customer [2].

\section{Simulation, simulation software and advantages}

In different literatures we can meet with more definitions, what is computer simulation. The most famous definition is it, where is written, simulation is virtual environment, which recalls real world or is same with him. This simple definition showing on simulation, where we need to create $3 \mathrm{D}$ model and simulate real conditions. In addition to such simulation is possible simulate different electrical schemes, which are interconnected and are able to start or shut down circuit. The output of the simulation is functional scheme, which is applied into device, which is intended for this.

The main part of simulation is software, which secured of simulation, contain all elements, which are connected with her or can contain library of elements. The most famous software in school environment is Matlab or superstructure in Matlab with same name Simulink. In general, the Matlab is programming environment specializing in scientific or technical numerical calculations, modelling, design of algorithms and so on. Superstructure Simulink is program, which is determined on simulating and modelling dynamical systems, which used algorithm of Matlab.

Environment of this software is similar as environment of basic text editor with little difference that used don't type text, but he inserted blocks, which represent some properties. Superstructure Simulink contains library of blocks, which are used for creation of simulation scheme.

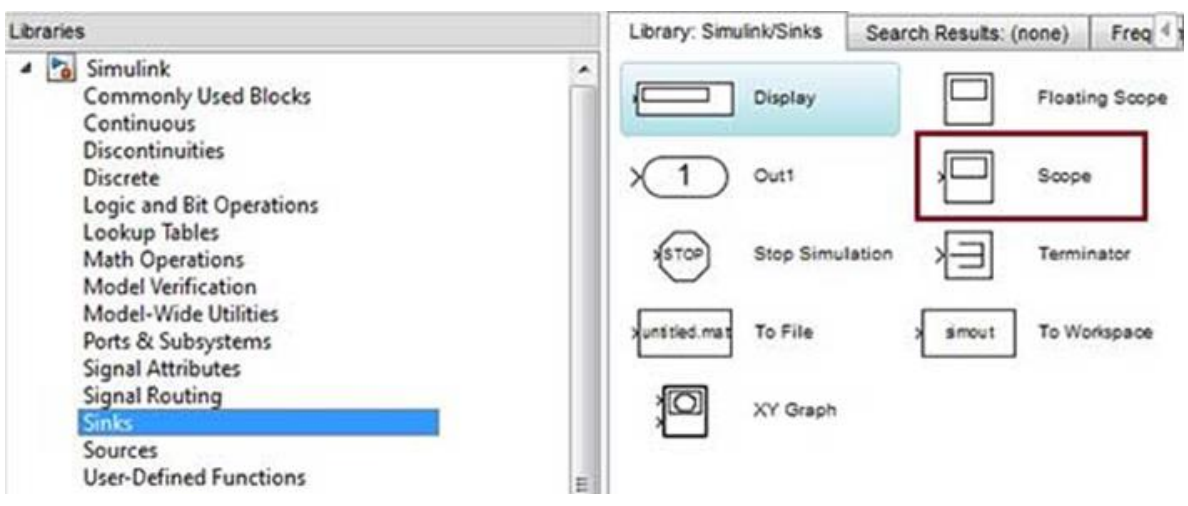

Fig. 1. Library in superstructure Simulink

As can be seen on Fig. 1 library is created very easily and elements aren't graphics intensive. Icons if this elements except graphical site, have functional site. On one or two sites they have small arrows. These arrows represent input or output to next device or element. Outputs of functional scheme can be graphs in different graphical styles, the 
behaviour of car tachometer, control traffic lights and the like. Outputs of this style has demanding graphic character and it is possible compare them with realistic devices [3].

The following software for simulation of manufacturing process is Flexsim, created by FSP Company. Company was created by Bill Nordgren in 1993. Former name of company was F\&H. In 1998, they developed first generation of 3D object-oriented simulation through Taylor ED graphic. First compact software with name Flexsim in version 1.0 was introduced in 2003. In this version of the software included 3D modelling environment and seamless integration of $\mathrm{C}++$ programming language. Animations can be displayed in three views $2 \mathrm{D}, 3 \mathrm{D}$ view and display through virtual reality [4].

FSP Company produces two types of software Flexsim Simulation and Healthcare Simulation. The second software is used for simulation of hospital premises and all things, which are connected with that. Flexsim Simulation is used primarily for simulation of manufacturing process. As already mentioned, the software is based on $3 \mathrm{D}$ object-oriented modelling so all elements are inserted through models. Working environment is similar as environment other software's. It contains status bar, working desktop, basic functions and library of elements how is shown on figure 2 .

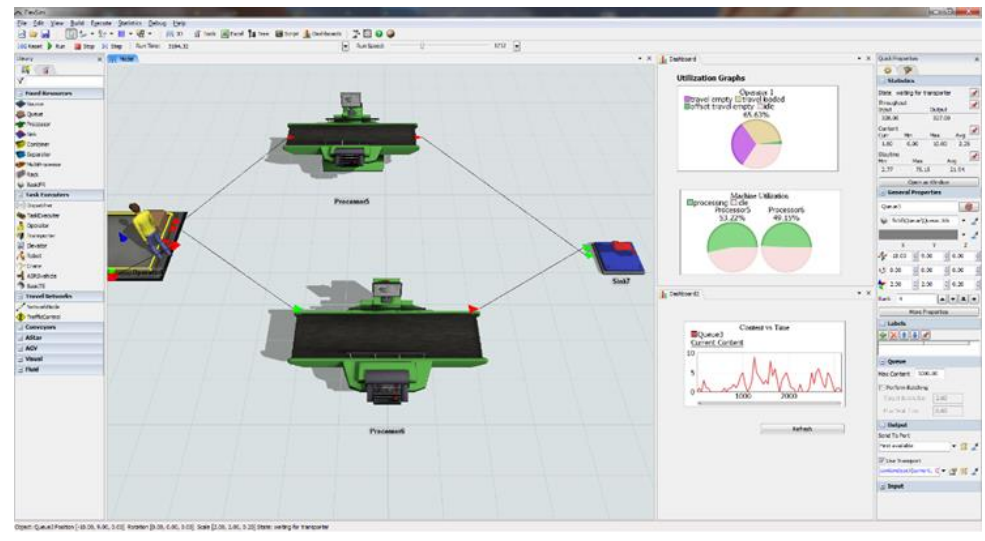

Fig. 2. Environment of Flexsim Simulation.

Modeling in software is simple, here is used drag and drop technique. Elements are inserted from library of elements. Between two elements are connecting lines, which represent connection of elements, for example: warehouse - machining center - conveyor manipulator - warehouse. This software exempting of the simple controls and optimizations. Advantage of software is option to insert 3D models from CAD systems. Except for models is possible import a drawings, which form the pattern of workplace. Next advantage are integrated scripts, which automatically respond on some elements, so it is not necessary to know programing language on high level.

Similar software as FlexSim is Witness from Lanner Group Company. The simulation software is created on base of object modeling, where predefined elements are inserted into scene. Basic elements represent warehouses, machining centers, manipulators and transport technology. Tools and machines can be found in library of elements, which are implemented in software. Library in addition to elements contain numerical or graphic components how graphs, time and piece units and variables, which are used for analytical part of simulation. This components are perceived how previous elements. One difference between numerical components and machines or warehouses is, that numerical components are not models.

Environment of Witness system is easy and uncluttered. On the top part are basic functions, on the second row are icons, which represent functions as connections, view 
change, zoom in, zoom out, text format, show and hide grid and more. In the middle part is work place, where is displayed grid. At the bottom part is library of elements, time displayed and icons for control of simulation. All windows can organize your own discretion (Fig. 3).

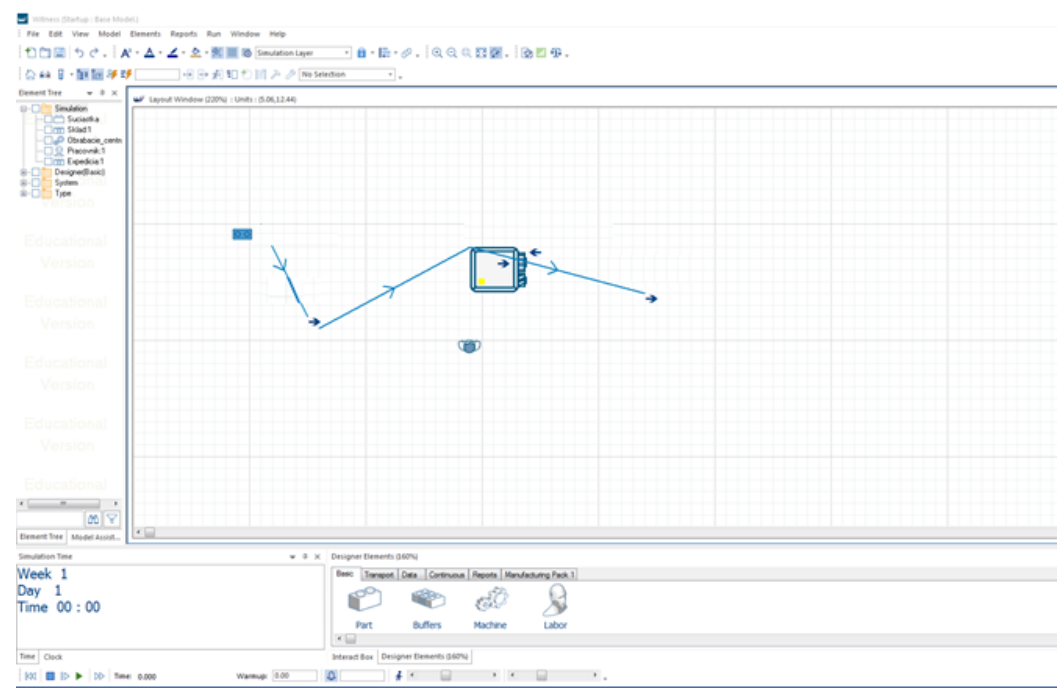

Fig. 3. Environment of Witness Horizon.

Compared to the previous simulation software Witness has better solved rules, where is possible to set for example, change length of the blank on input and output. It is more modular, has a lot of output options, display options and so on.

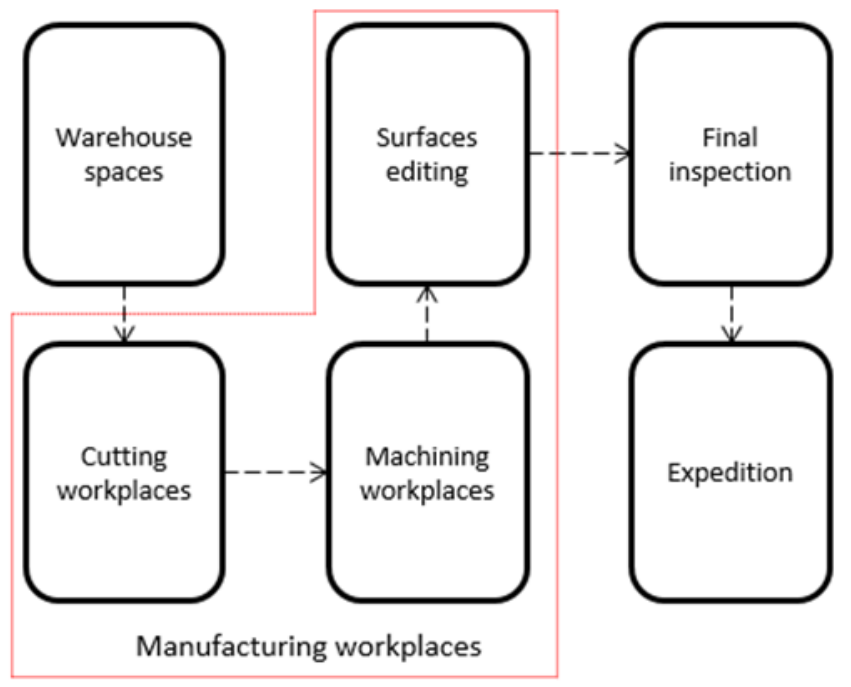

Fig. 4. Basic scheme of manufacturing process.

On Fig. 4 is displayed basic scheme, which points to process blank in its production. First workplace are warehouse spaces, where are saved blanks, which are designated for next processing. Through transport devices, the blanks are transported into manufacturing hall, which contain three cutting workplaces, machining workplace and surfaces editing. 
Since those three workplaces are in one manufacturing hall, the best a means of transport is conveyor. At the end of manufacturing process is inspection, where are all parts inspected. If some part contain defects, so they go to waste. The last workplace is expedition, where the packing materials occurs and the temporary storage.

This basic scheme does not contain count of machines and tools, which are used in simulation, but serves as informative character for create real simulation, which contain all parts.

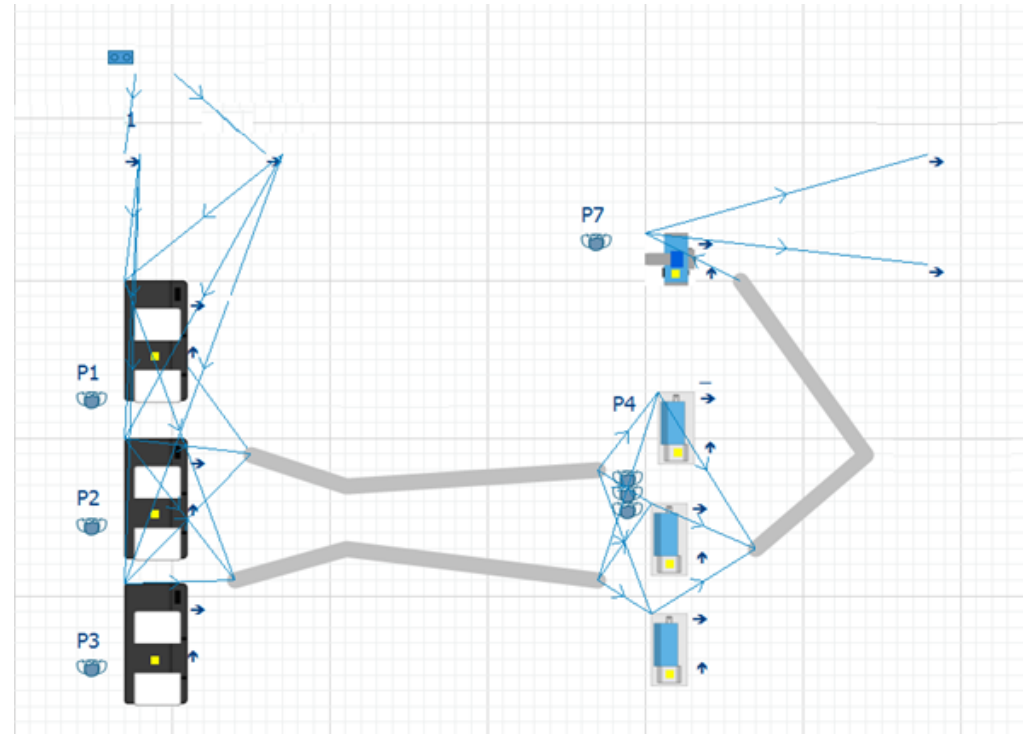

Fig. 5. 2D scheme of workplace.

On Fig. 5 are displayed elements of simulation, which represent real machines and devices. Blue lines in scheme represent connections between elements. Through connections, simple rules as "PUSH to" or "PULL from" are created automatically. Except to these simple rules in simulation are rules, which determine how many percent of components must go into machines or warehouses. In input or output rules are include change of blanks as length, weight and etc. On the start, the blank has the largest size, because he was not in manufacturing process yet. These changes are not include as rules, but as changes on start or end in workplaces. Changes are still identical at the end of first workplace and on the beginning of the second workplace, since the state of the blank between two workplaces is still similar.

In rules of machines are included changes or tool wear, which occurs at a certain time. Except for wear of material, it is need to be reckoned with withdrawals and deposits blanks into and out from machine. These rules are not intended to specifically relationships. They are expressed as settings of machine or work pause of machine.

For machines, which are used for transport of parts the main condition is capacity of machine. The next condition is type of transport as conveyor or rail transport and etc. If you use conveyor, so you need choose type of conveyor, velocity and dimensions. It is possible to choose settings, where length of conveyor is the same of capacity [5].

Important element of simulation are works, who care about a tools change, blanks and collecting of finished pats and moving on transport machines. 


\section{Flow material in simulation}

At the beginning of the simulation are the blanks, which are heading directly into two warehouses with names warehouse 1 and warehouse 2 . Warehouses have capacity 50 pieces of blanks. The blanks enters into warehouses from transport in interval 1 minute about the number 10 pieces. After this, blanks heading directly into cutting workplace. Capacity of one cutting center are 4 blanks. Cutting takes 5 minutes. Cutting workplace contain three machines, so in 5 minutes workplace can produce twelve blanks, who are intended for further processing.

\section{Number of output pieces}
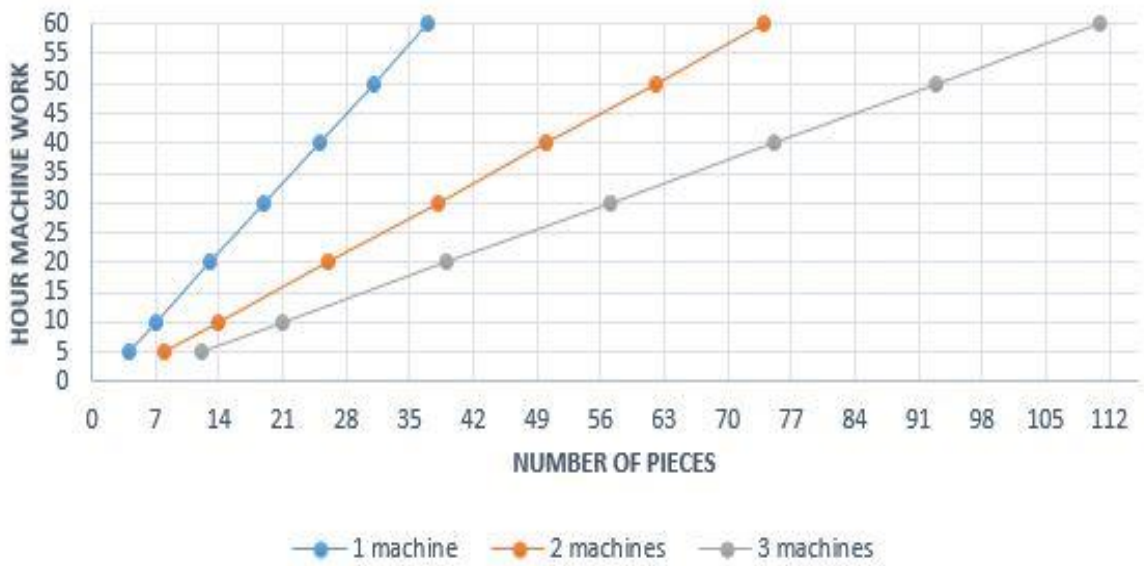

Fig. 6. Number of pieces on cutting workplace.

Fig. 6 describes the effectiveness of the use of multiple machines in the workplace. For one hour, one machine can produce 37 pieces of blanks, which are determined for next processing. If we increase the number of two cutting centers on workplace, so productivity will be 111 pices for one hour. For cutting of blanks is created the loss, which is one respectively three blanks for 5 minutes. The loss for one hour is 33 blanks. It is caused by choosing blanks from centers, moving on conveyors and delivering another blank into cutting center. In cutting center is used one type of tool. In the production of a large number of occurs tool wear. Tool without loss quality of cut can create 111 similar operations. If we use tool for more operations, so on material are created defects, which reduce quality of cut. In that case it is necessary to clean the reservoir of excess material and splinter. Cleaning takes 5 minutes on every workplace. This operation takes place every three hours, also at 8 hour working time to occur 2 times. The total downtime of the machine is in this case 10 minutes.

From first workplace, blanks go through conveyor to another workplace. Capacity of conveyors is max 60 pieces of blanks. If follows that conveyor are equally burdened. It is not possible for one conveyor was idle. Blanks don't have their place on conveyor, but are amassed if the conveyor is not full. The two conveyors operate in parallel. Time of transmission components is 3 minutes from insertion components on conveyor.

The following workplace is machining workplace, where are $3 \mathrm{CNC}$ enters. In these centers occurs components working before final form and surface working. Operations who are carried out on machines takes two and half minutes. Operations are created by two tools (roughing and surface planning). The blank is circular form and it is lathed from one and second sides. Inserting blanks into machine, rotating and removing of part takes 20 
seconds. Tool change pending automatically through rotation of tool container. This cycle takes 5 seconds. The analysis shows that all times that are related to wear and tear lasts 35 seconds.

\section{Efficiency of the use of multiple CNC} centers

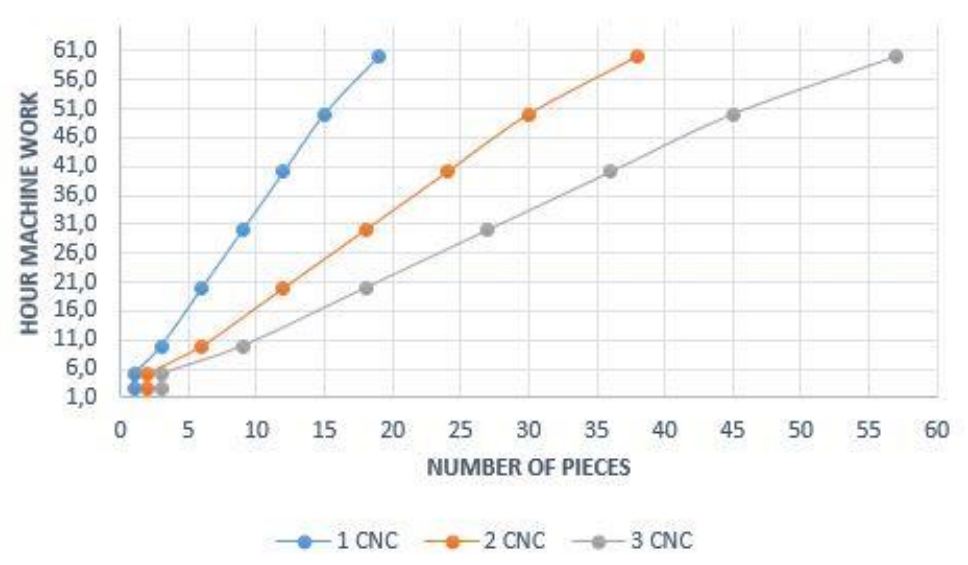

Fig. 7. Number of pieces on machining workplace.

How possible see on Fig. 7, for more complex operations is lower machine productivity. One machining center can produce only 19 pieces of finished products. After calculating working time and pause of machine, which are necessary for good machining time and pause of machine, which are necessary for a good machining of blanks, clean working time of machine is 57 minutes and 45 seconds. When using more CNC machining centers, productivity is 3 times bigger. Working time is the same, since the all machines works in same time. Least effective time is first 5 minutes, when machine needing for production of 2 pieces parts 6 minutes and 30 seconds.

Next stop for finished parts is surface editing. Transport from one to second workplace is through conveyor too. Type of conveyor is similar than conveyor between preceding workplaces. It is roller conveyor. Capacity of conveyor is 60 pieces of parts. Conveyor is still in move. After machining, part is transported to next workplace. It is not possible to be conveyor was full, because parts are automatically sorted from conveyor. Conveyor is full routinely.

Last workplace in manufacturing process is surface editing of parts. In this workplace is only one machine, which able to work with a lot of parts in same time. Container in machine has capacity 100 pieces of parts. Filling one container in average of take 2 hours. These time data are bound on previous workplaces. If be previous workplaces had more machines, so productivity would be bigger. Capacity of conveyor or velocity should be bigger too. 


\section{Effectiveness of the surface treatment plant}

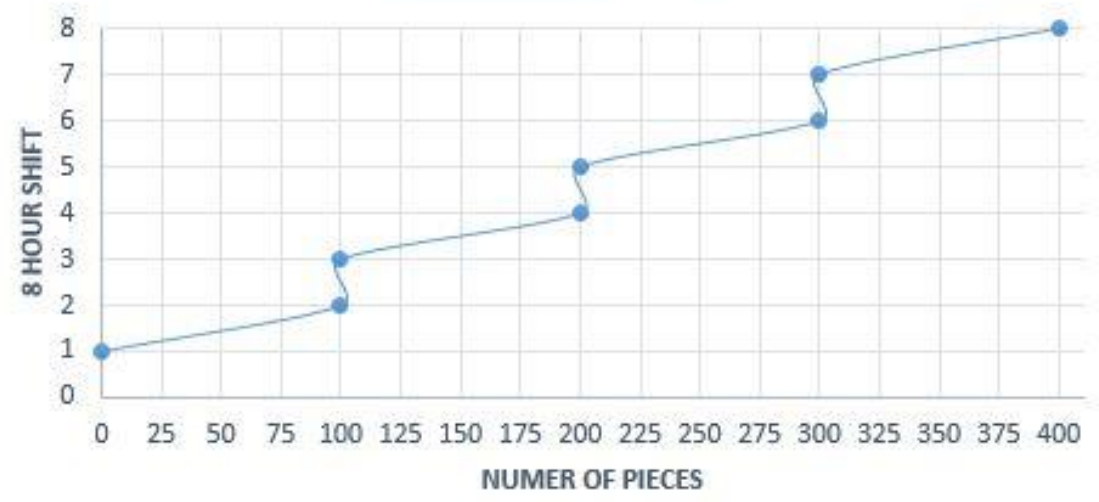

Fig. 8. Productivity of surface editing workplace.

Workplace on surface editing can work with a large number of parts. Hos is displayed on Fig. 8, workplace produce the first parts after 3 hours work. Every next hour has similar data about pieces, because it is caused by processing. Two hours is time, which is necessary for filling of container. This time is measured from beginning manufacturing process until to inserted parts into container. In next hour this data are lower, because work time of machine is 60 minutes. Surface editing takes 50 minutes on all parts. Last 10 minutes is control, which should recognize defects on parts. From two hours waiting on next container, time is reducing on one hour. From picture is possible to evaluate, two hours from beginning of process is machine non-productive. Idle time of machine is on first process, because container is full on the end of last shift. It appears from this that machine can work from begin of day. In this case, machine can produce about 100 pieces of parts more.

Final part in all process is warehousing in temporary storage, which is possible to name a expedition. Even before a certain percentage is moving into storage space with scraps.

\section{Conclusion}

Simulation of manufacturing process is good tool for data analysis, which taking place in company. Through simulation is possible to watch all areas in company, who are producing some product. In our simulation was created workplace, which contain three manufacturing areas. On these workplaces were monitored a productivity and efficiency of work based on the number of machines. After final calculation was possible detect inefficiency of workplaces.

From the point of productivity best workplace was the last, it was surface editing of products. Workplace includes machines, which can work with a great deal products at the same time. Machines are no productive three hours every day. It is caused by past operations and transport, where product will come after one hour from begin of manufacturing process. These idle time it was possible to eliminate increasing the number of pieces of transport equipment or increase the number of pieces of machinery in the workplace.

Second workplace has balance effectiveness. On the workplace don pt arise idle times in manufacturing process. For the improvement of workplace quality be possible add one or 
two machines. Second idea is add more conveyor between workplaces or change type and capacity of conveyor.

First workplace has main task in all process. It starting up work on next workplaces. In the case of damaging it is the whole process stopped. Machines, which are on workplace can work with more blanks in same time. In such work takes place more rapidly a tool wear. It is necessary change tool more often. As with the other work efficiency could be increased by adding new equipment or changing technology that is used.

The overall efficiency of the workplace can be improved in various ways. Whether it is a purchase of new machinery and their inclusion in the production or switch to a different technology depending on the requirements of entering as master data in the enterprise. These aspects can simulate and through simulation to create realistic looking to invest in an established company.

\section{References}

1. T. Prestero, Verification of a six-degree of freedom simulation model for the REMUS autonomous underwater vehicle, MIT, California, USAm (2001)

2. D. ATHOW, http://www.techradar.com/news/computing/best-workstation-the-bestenterprise-desktop-for-you-1271279 (2017)

3. M. Tothová, J. Pitel', SISY 2014, IEEE, 209-214, ISBN: 978-1-4799-5997-6 (2014)

4. W. B. Nordgren, WSC '03, Proceedings of the 35th conference on Winter simulation: driving innovation (2003)

5. M. Andrejiova, A. Grincova, D. Marasova, Wear. 368, 400-407, http://www.sciencedirect.com/science/article/pii/S0043164816304616 (2016), 
